\title{
Adjacent, Adherent, Invaded: A Spectrum of Biologic Aggressiveness Rather Than a Rationale for Selecting Organ Resection in Surgery of Primary Retroperitoneal Sarcomas
}

\author{
Dirk C. Strauss ${ }^{1}$, Salvatore L. Renne ${ }^{2}$, and Alessandro Gronchi ${ }^{3}$ (i) \\ ${ }^{1}$ Department of Surgery, Royal Marsden Hospital NHS Foundation Trust, London, UK; ${ }^{2}$ Department of Pathology, \\ Fondazione IRCCS Istituto Nazionale dei Tumori, Milan, Italy; ${ }^{3}$ Department of Surgery, Fondazione IRCCS Istituto \\ Nazionale dei Tumori, Milan, Italy
}

Complete surgical resection of retroperitoneal sarcoma (RPS) remains the only potential curative treatment. The surgical strategy to achieve this optimal and most appropriate resection for each patient still creates some contention and controversy. Most contentious in this debate is the approach to the organs and structures neighboring a retroperitoneal sarcoma, with surgical strategy covering a spectrum from a conservative approach of organ-preserving and simple excision of the tumor alone to resection of the tumor and contiguous organs only when evidence of direct involvement exists to liberal multivisceral compartmental or extended resection of contiguous organs even if uninvolved. In the future, as more information is collected in a standardized and prospective fashion from specialist sarcoma units, this debate should evolve to define the most appropriate and personalized strategy, including extent of surgery, for each patient based on all available patient and prognostic factors.

In this issue of Annals of Surgical Oncology, the group from Dana-Farber/Brigham and Women's Cancer Centre ${ }^{1}$ report their experience of selective organ resection for primary retroperitoneal sarcomas, with the objective not to define the appropriate approach to surgical resection, but rather to examine the rate and rationale for organ resection and the rate of histopathologic organ infiltration (HOI) according to histologic subtype. In this elegant

(C) Society of Surgical Oncology 2017

First Received: 7 September 2017;

Published Online: 27 October 2017

A. Gronchi

e-mail: alessandro.gronchi@istitutotumori.mi.it retrospective study, Fairweather et al. propose a six-tier system for classifying the rationale for organ resection.

The rationale for organ resection was determined by reviewing the historic description of the procedure from the operative report. In this study, HOI is defined as infiltration of organ parenchyma or vessel wall by tumor cells. Organs that appeared to be inseparable from tumor were considered adherent. In 99 patients, at least one organ was resected, and the rationales for organ resection most often reported were tumor adherence, organ encasement, involved end-organ vasculature, and suspected invasion or tumor origin.

This study has some limitations. First, the retrospective nature of the study makes it difficult to obtain a clear and accurate rationale from historic operative reports of organ resection. A patient selection bias existed regarding organ resection (e.g., influenced by age and comorbidities of the patient, surgical aggressiveness of the surgeon) and the degree of intraoperative subjectivity when a decision was made regarding the presence of a visible dissection plane or whether organs were adjacent or adherent to tumor. The study also does not include preoperative radiologic analysis, which should also contribute to the surgeon's decisionmaking process around the necessity for organ resection.

The study lacked a standard for specimen processing, margin sampling, and characterization and definition of organ invasion. Consequently, the definition of HOI was not inclusive of tumors adherent to organs, which perhaps involved perivisceral/capsular invasion but not necessarily organ parenchyma infiltration. In many cases, although no parenchyma infiltration occurred, negative margins could be achieved without resection of the organ (Fig. 1). 


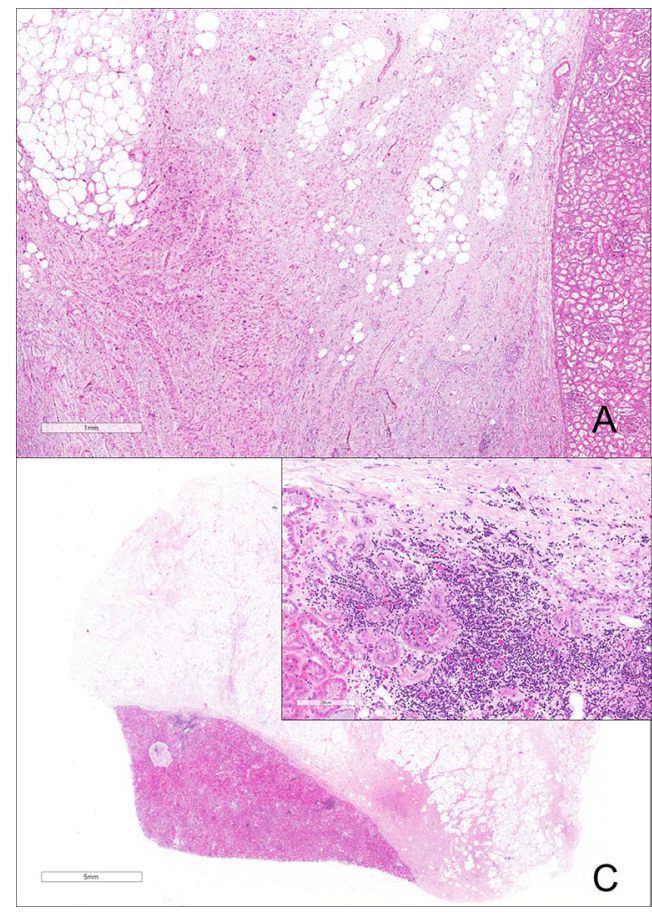

FIG. 1 a Dedifferentiated liposarcoma invading the perirenal capsule without invasion of the renal parenchyma (formally, the organ is not invaded, but no way exists to separate the tumor from the organ other than doing an intralesional resection). b Well-differentiated liposarcoma invading the perivisceral colonic fat (again, as in

Mussi et al. ${ }^{2}$ also reported on HOI after extensive serial sections of the sarcoma and involved viscera had been performed. They defined visceral involvement by tumor as two major types: (1) the infiltrative type, in which the visceral structure is not embedded by the tumor but has clear, either focal or diffuse, infiltration by nests or single cells of normal renal cortex, hilar vessels, tunica muscularis of the bowel and the ureter, psoas muscle, pancreas, adrenal gland, liver, spleen, and the like, and (2) the pushing type (expansive), in which the organ is totally or partially embedded in tumor with ill-defined borders but no microscopic evidence of infiltration (i.e., invasion of perirenal adipose tissue by well-differentiated liposarcoma (WDLPS) without infiltration of the cortex). These authors found that 92 of 151 organs resected were involved by the tumor $(60.9 \%)$. This contrasts with the current study, which reported HOI in only 77 (25\%) of 302 organs resected. Differences in pathologic sampling and defining of HOI must account for this reported variance in HOI.

Mussi et al. ${ }^{2}$ further reported a difference according to histologic subtype. The infiltrative pattern was more often observed in leiomyosarcoma and non-lipogenic tumors, whereas the expansive pattern was more often observed in liposarcoma.

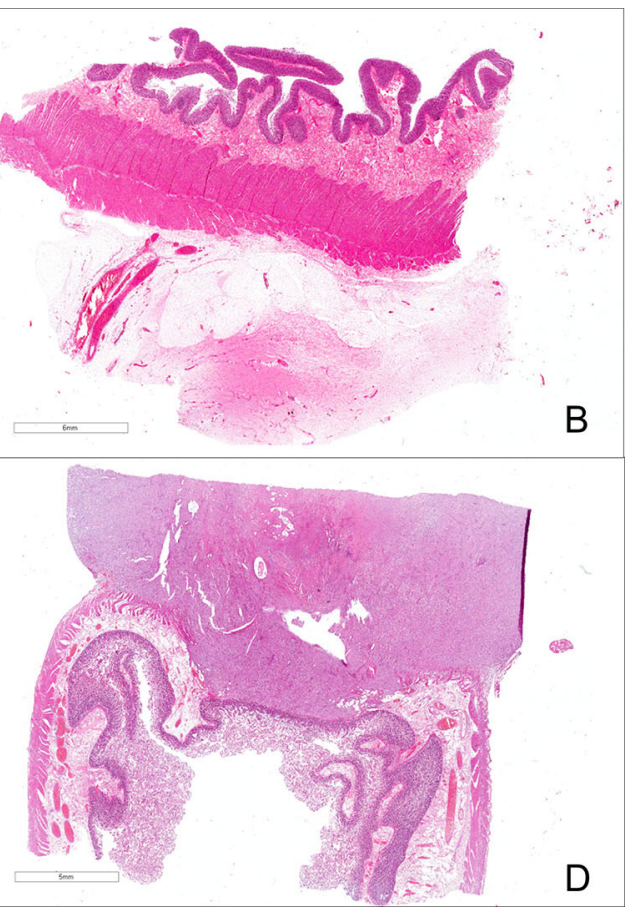

a formally, the colon is not invaded, but sparing the colon would significantly compromise the quality of the surgical margins). c Dedifferentiated liposarcoma invading the renal parenchyma. d Dedifferentiated liposarcoma invading the wall of the colon

The authors state that the current study aimed to understand better the rationale for individual organ resection and to examine HOI among resected organs, which in turn may better define an appropriate minimal extent of contiguous organ resection. The dilemma, however, is that invasion of the parenchyma or the perivisceral/capsular zone cannot always be anticipated on either preoperative imaging or intraoperative evaluation. Therefore, perceived absence of histopathologic invasion is not an argument to preserve organs. This is supported by the finding reported in this study that among all the patients, HOI was present in $19 \%$ of the organs resected due to tumor encasement and in $26 \%$ of the organs adherent to tumors, even when organ invasion was not suspected intraoperatively. Among the patients with dedifferentiated liposarcoma (DDLPS) who underwent a colonic resection, 33\% had confirmed HOI, none of which were suspected intraoperatively. Their report has a selection bias in the decision to resect a given organ, which limits generalizability of the study results. For instance, only two organs were removed for suspected invasion of WDLPS, and HOI was confirmed in both. The rate of HOI in the organ resected may be known and analyzed extensively, but the unknown in this equation remains the organs with HOI that were not resected and may harbor microscopic residual tumor. In the light of 
these limitations, indications such as "nephrectomy may not be necessary for WDLPS" cannot be fully supported by the results of this study.

In a recent report from Fondazione IRCCS Istituto Nazionale dei Tumori-Milan, ${ }^{3}$ presented at the American Society of Clinical Oncology annual meeting 2017, in which visceral invasion was investigated after extensive sampling, visceral infiltration was classified as infiltration of perivisceral fat, early infiltration (i.e., renal/adrenal capsule, muscular fascia, contact with muscular tunica of hollow viscera), and infiltration of the viscera. Organ infiltration to some extent was seen in $80 \%$ of the patients undergoing liberal multivisceral resection for primary RPS. Visceral resection margins were positive in $12 \%$ of the patients. After adjustment for known prognostic features (patient age, tumor histology, grade, and size), the patients with negative visceral resection margins and positive visceral infiltration exhibited a worse overall survival (OS) than the patients without organ infiltration.

Organ invasion by sarcoma should be seen as a marker of biologic aggressiveness rather than as a reason for resecting or not resecting an organ. The group from DanaFarber/Brigham and Women's Cancer Centre also has previously reported on the adverse prognostic implications of histolopathologic organ invasion. ${ }^{4}$

The concept of liberal extended compartmental resection has caused much debate in recent years. This surgery for retroperitoneal sarcomas aims to minimize marginality and achieve negative resection margins whenever possible, at a cost of resecting adherent and sometimes adjacent organs, even if not overtly infiltrated. The surgical concept advocated is a policy of more liberal visceral en bloc resection that includes an envelope of normal tissue and uninvolved adjacent organs around the tumor in certain dimensions to minimize the risk of microscopic positive margins, with reports of improved local control and overall survival. ${ }^{5}$ The technical details of this approach have been published to establish a standardized surgical strategy. ${ }^{6}$

A radical surgical approach, especially with regard to "disposable organs," is safe and associated with low morbidity when performed at a specialist sarcoma center. ${ }^{7}$ Complete surgical resection with clear margin status is the only potentially modifiable factor that can be optimized to offer the patient the best chance of cure at the time of primary surgery. High-risk resections (i.e., pancreaticoduodenectomy) should be carefully considered on an individual basis, weighed against anticipated disease biology, and never performed on a routine basis. Judgment must be used in deciding which adjacent viscera and vital structures to sacrifice, considering the potential benefit in local control against the inherent tumor biology, surgical morbidity, and ultimate quality of life.
The clinician is unwise to compromise the quality of local treatment during the first operation, which offers the best opportunity to perform the only operation that may determine the ultimate outcome, in order to preserve organs for the purpose of facilitating potentially systemic treatments in the future that have no overall survival benefit. Extended surgical resection has acceptable morbidity and is required as primary treatment to offer the best opportunity of cure because tumor recurrence is mostly incurable for the patient. This is all the more true for tumors with a predominant local recurrence risk, typically WDLPS and grade 2 DDLPS. ${ }^{5}$

We agree with the authors that appropriate operative planning requires, at a minimum, resection of organs with significant risk of tumor involvement. The open questions remain: what equates a significant risk and how does one judge whether tumor involvement exists? Retroperitoneal sarcoma is not a single disease, and the spectrum of different histologies is variable in terms of growth pattern and pattern of failure.

During the last few decades, a large amount of understanding has emerged from collection and analysis of information from large prospectively maintained databases of patients with primary retroperitoneal sarcomas. ${ }^{8}$ Differences in biologic behavior, microscopic growth pattern, and patterns of disease recurrence according to histologic subtype have become clearer. Given this different pattern of growth and locoregional risks, the rationale for organ resection may rather be based on histology subtype than on a macroscopic assessment of direct organ invasion. Extended resection with liberal multivisceral resection of adjacent organs for high-grade leiomyosarcoma will have a negligible impact on overall survival because most deaths are related to metastatic spread, and the risk for local recurrence is only 5-10\%. Leiomyosarcomas exhibit a more infiltrative growth pattern, in which surrounding involved organs either are microscopically separate or, when involved, have a visceral structure not embedded by the tumor but involving a clear, either focal or diffuse, infiltration by nests or single cells.

In contrast, the metastatic risk for WDLPS is effectively nil and less than $10 \%$ for grade 2 DDLPS, whereas most deaths are related to locoregional failure. The growth pattern found in liposarcomas is more often a pushing type (expansive), in which the surrounding viscera/structure may be totally or partially embedded in tumor with illdefined borders, but without microscopic evidence of organ infiltration. $^{2}$ Although organ infiltration may not be evident, surgical resection of these adjacent uninvolved organs will provide a better quality of margin, whereas attempted dissection of an embedded organ from the tumor surface risks leaving microscopic residual tumor and compromising local control. 
A limitation of this reported study and similar studies from other specialist sarcoma centers is lack of a standard for specimen processing, margin sampling, and characterization and definition of organ invasion. The Transatlantic RPS Working Group (TARPSWG.org) is a multi-institutional international collaboration of specialist sarcoma centers from Europe and North America established to generate a pooled data set of primary RPS patients as a means of overcoming the rarity of this family of diseases. From this collaboration has followed the creation of a virtual platform for prospective collection of standardized patient clinical information, radiologic imaging, surgical information, pathologic results, data on tumor biology, and a quality of life (QoL) evaluation questionnaire from multiple reference institutions. In an attempt to standardize reports, the TARPSWG can organize consensus on surgical findings and decisions around adjacent organ resection, as well as on pathology processing and reporting of margin/ organ invasion. By collecting these data in a standardized and prospective fashion, the project hopes to identify clinical, radiologic, and pathologic characteristics that may influence the management and oncologic outcome for this rare collection of tumors. All institutions with an interest in RPS are invited to join!

DISCLOSURE There are no conflicts of interest.

\section{REFERENCES}

1. Fairweather M, Wang J, Jo VY, Baldini EH, Bertagnolli MM, Raut CP. Surgical management of primary retroperitoneal sarcomas: rationale for selective organ resection. Ann Surg Oncol. 2017. doi:10.1245/s10434-017-6136-4.

2. Mussi C, Colombo P, Bertuzzi A, Coladonato M, Bagnoli P, Secondino S, et al. Retroperitoneal sarcoma: is it time to change the surgical policy? Ann Surg Oncol. 2011;18:2136-42.

3. Renne SL, Tagliabue M, Pasquali S, Collini P, Barisella M, Callegaro D, et al. Prognostic value of microscopic evaluation of organ infiltration and visceral resection margins (VRM) in patients with retroperitoneal sarcoma (RPS). J Clin Oncol. 2017;35:11074. doi:10.1200/JCO.2017.35.15_suppl.11074

4. Fairweather M, Wang J, Jo VY, Baldini EH, Bertagnolli MM, Raut CP (2017). Incidence and adverse prognostic implications of histopathologic organ invasion in primary retroperitoneal sarcoma. J Am Col. Surg. 224(5):876-883.

5. Gronchi A, Miceli R, Colombo C, Stacchiotti S, Collini P, Mariani $\mathrm{L}$, et al. Frontline extended surgery is associated with improved survival in retroperitoneal low- to intermediate-grade soft tissue sarcomas. Ann Oncol. 2012;23:1067-73.

6. Bonvalot S, Raut CP, Pollock RE, Rutkowski P, Strauss DC, Hayes AJ, et al. Technical considerations in surgery for retroperitoneal sarcomas: position paper from E-Surge, a master class in sarcoma surgery, and EORTC-STBSG. Ann Surg Oncol. 2012;19:2981-91.

7. MacNeill AJ, Gronchi A, Miceli R, Bonvalot S, Swallow CJ, Hohenberger $\mathrm{P}$, et al. Postoperative morbidity after radical resection of primary retroperitoneal sarcoma: a report from the Transatlantic RPS Working Group. Ann Surg. 2017. doi:10.1097/ SLA.0000000000002250.

8. Gronchi A, Strauss DC, Miceli R, Bonvalot S, Swallow CJ, Hohenberger $P$, et al. Variability in patterns of recurrence after resection of primary retroperitoneal sarcoma (RPS): a report on 1007 patients from the multi-institutional collaborative Transatlantic RPS Working Group. Ann Surg. 2016;263:1002-9. 